\title{
$\begin{array}{ll}\text { Ie portiQue } & \text { Le Portique } \\ \text { Revue de philosophie et de sciences humaines }\end{array}$ \\ e-Portique | 2006
}

\section{Le soin de toucher}

Bernard Andrieu

\section{OpenEdition \\ Journals}

Édition électronique

URL : https://journals.openedition.org/leportique/867

DOI : 10.4000/leportique.867

ISSN : 1777-5280

\section{Éditeur}

Association "Les Amis du Portique"

\section{Référence électronique}

Bernard Andrieu, "Le soin de toucher », Le Portique [En ligne], e-Portique, mis en ligne le 08 janvier 2007, consulté le 11 septembre 2022. URL : http://journals.openedition.org/leportique/867 ; DOI : https://doi.org/10.4000/leportique.867

Ce document a été généré automatiquement le 11 septembre 2022.

Tous droits réservés 


\title{
Le soin de toucher
}

\author{
Bernard Andrieu
}

1 Si le toucher est à l'origine même de la relation thérapeutique, le toucher est devenu un nouveau style de communication sociale et communautaire du soi. Dans sa sociologie de la post modernité Michel Maffesoli décrit, dès 1993, combien le style tactile participe d'une transmutation des valeurs par un autre rapport à l'altérité : dans une société concrète, d'action en commun " l'autre est celui que je touche et avec lequel je fais quelque chose qui me touche" ; la recherche de l'ambiance émotionnelle dans des activités affectuelles comme l'écologie, la musique, la solidarité humanitaire. Ainsi “ il peut y avoir une communication qui n'ait pour seul objectif que de " toucher" l'autre, d'être, simplement, en contact, participer ensemble à une forme de grégarité" ${ }^{2}$

Communiquer par le toucher

2 Le développement des biojoux technologiques (sextoys ${ }^{3}$, gode ${ }^{4}$, greffes, implants, transcorps $\left.s^{5} . ..\right)$ que l'on peut incorporer en soi de manière éphémère ou définitives définisse une sensualité fondée sur un mieux être, une recherche hédoniste et une pratique tactile échangiste: le corps incorpore des biojoux ou plutôt les biojoux deviennent le centre autour duquel une recomposition esthésiologique s'effectue tant du point de vue du vécu que de celui du vivant. L'incorporation du biojou rend moins le corps dépendant de sa technologie mais vient renouveler l'expérience de soi, des autres et du monde extérieur.

3 L'externalité sensible précipite le sujet dans la recherche des sensations tangibles, faute de trouver en soi, dans sa mémoire et dans ses expériences, les repères suffisants pour évaluer avant la prise de risque l'intensité, la nature et l'orientation du contact. L'intériorité tactile se constitue ainsi par l'externalité tangible sans que le tactile puisse conserver l'intensité du tangible. Cet affadissement du tangible dans le tactile nourrit la nécessité d'intensifier l'expérience sensorielle par un renouvellement indéfini des objets tangibles. Le sujet tactile ne parvient jamais à l'identité fusionnelle avec l'objet tangible.

4 De cet écart naît la recherche indéfinie du tangible sans la proportion ou la mesure susceptible de contenir le tangible dans le tactile. Le sujet tactile a alors la tentation de devenir un sujet tangible : c'est-à-dire de vivre l'intensité sensorielle comme une 
succession d'instants dont la mobilité nous ferait ressentir immédiatement la matière. Aucune substantialité du sujet n'assure ici une permanence et une conservation de l'expérience tangible, le sujet voulant devenir sensible par la sensation, se touchant en touchant. La spirale tangible se met en place dès lors que le sujet devient dépendant de son externalité pour éprouver de nouvelles sensations tactiles intérieures. L'augmentation des prises de risques, l'intensification du contact avec des variations de plus en plus fortes vers la violence sur l'objet ou vers l'invasion de l'objet sur/dans le corps du sujet trahit un manque d'apprentissage susceptible d'évaluer la prise de contact.

La peur du toucher

5 Face à la sécurisation et la télésurveillance des espaces publics ${ }^{6}$, le repli domotique ne suffit pas à éduquer au toucher renfermant chacun/e devant les écrans plasma d'une télévision numérique à la carte. Le home-cinema remplace le cinéma privatisant l'image dans une jouissance de salon sans l'expérience de l'émotion collective et partagée. L'individu onaniste jouit à travers des sites virtuels du plaisir exhibitionniste sans toujours parvenir à rencontrer d'autres corps que le sien. La vie sans sexe devient une tentation et une impasse.

6 L'affaire d'Outreau a révélé l'impact du discours des enfants construits en accusations pédophiles à partir d'une interprétation des images pornographiques et autres objets sexuels utilisés par le monde adulte. Ce pouvoir tactile des images a fait accroire en l'authenticité de la parole enfantine tant derrière les scénario récités se dressaient un imaginaire sadique d'instrumentalisation du désir. Le toucher violent reste l' arrière plan fantasmatique d'une expertise structuraliste qui ne sait plus distinguer dans le langage l'effraction, le viol ou la torture. La dénonciation calomnieuse, l'affaire Baudis ${ }^{\circ}$ le prouverait, suffit à dérégler le jugement en transportant le toucher dans les mots, en provoquant la représentation impossible de la prostitution $^{10} \mathrm{du}$ politique, de la pédophilie du notable ou de la torture des enfants.

7 Pourtant la violence physique est bien réelle et spécialement mise en scène par le media pour provoquer l'évènement collectif de manifestations de victimes et de personnes compatissantes aux crimes. Au service d'une sécurisation sarkosienne du social, l'arbre du crime crapuleux sur des victimes démunies occulte la forêt des violences envers les femmes ${ }^{11}$; la dégradation du lien, même pédagogique, s'exprime dans l'atteinte du corps professoral ; la peur d'être touché, sinon atteint, précipite deux jeunes français dans l'électrocution, avant que le langage républicain de " racaille » n'enflamme en touchant le corps de banlieue.

8 Le corps de l'immigré, brûlé dans les incendies racistes des squats, parqué dans un gymnase de Cachan ou déporté vers ce qui seraient leur pays d'origine entretient l'idée que rien ne devrait toucher à la pureté de notre corps national.! Quand on a plus que son $\operatorname{corps}^{12}$, le toucher pour les exclus du capital reste une expérience fragile mais nécessaire pour sentir et se sentir. Même l'insulte désormais nous touche car le langage est corporel, constitués que nous sommes des caresses verbales, des regards touchants et des mots adressés

Le soin de toucher

9 Le développement des $\mathrm{SPA}^{13}$ et autres lieux du soin corporel du soi ${ }^{14}$ constitue une véritable alternative à la médecine chimique et mécanique en retrouvant dans le geste une anthropologie sensorielle ${ }^{15}$. Le massage ${ }^{16}$ doit conduire au de-stress ${ }^{17}$ par la libération 
intérieure de la peau en traversant ses couches pour délivrer le sujet de ses pesanteurs psychiques. La croyance holiste renouvelle, en retrouvant les traditions hindouistes et orientales si exaltées par notre revival actuel des années 70 , le soin thérapeutique: toucher devient le moyen de se sentir et de ressentir le vivant de notre chair si anesthésiée par l'illusion visuo-tactile de la téléréalité

L'érotisme $^{18}$ expérimente cette alternative tactile jusqu'à la violence consentie du SM, ${ }^{19}$ ou en se maintenant dans l'immobilité du bondage ${ }^{20}$. Les sex toys modifient le contact en introduisant les objets et les jouets ${ }^{21}$ dans son corps et le corps de l'autre, révélant des technologies du genre dans le couple corporel. Inverser les conventions et les rôles ouvre la possibilité d'une cruelle zélande ${ }^{22}$ lesbienne d'épilation. La partouz révèle que toute notre réalité devient " pornotransformable ». De Putain à Folle, Nelly Arcan subit son avortement en découvrant le corps intérieur de l'amour perdu $u^{24}$. Toni Bentley ${ }^{25}$ affirme enfin la sodomie comme une expérience tactile irremplaçable qui découvre le trou du corps, le corps comme un trou sans fond. Lucie Lux ${ }^{26}$ exalte la masturbation moins comme un retour en soi que comme une moyen de ne plus s'ennuyer?

11 Prendre soin de soi et des autres trouvent dans l'accompagnement palliatif des mourants et dans l'éducation sensorielle des enfants des modes d'apprentissage. Refusant ces cours intérieures d'un corps boîte d'aluminium ${ }^{27}$, souffrir n'est plus qu'une affaire de contrat ${ }^{28} q u^{\prime}$ 'il conviendrait de dénoncer, sauf le respect que l'on nous doit ${ }^{29}$. Toucher à son hybridité

12 Chacun et chacune veut aujourd'hui se composer un corps à soi ${ }^{30}$. Un corps à soi est la possibilité de se construire une identité en utilisant les progrès technoscientifiques qui transforment les données naturelles. L'hybridation consiste ici à introduire dans son corps des produits, des outils, des machines qui donnent une nouvelle identité: l'identité hybridée n'est plus l'identité naturelle dont nous avons héritée de nos parents... ce corps d'identité est composite, mélangée et hybridée comme en témoigne les piercings, les prothèses, les implants, les rôles sociaux, les genres et postures...Mais ces hybrides ne sont pas à comprendre comme extérieurs au corps, ils sont incorporés en nous par nos savoir-faire, nos habitudes et nos techniques du corps. Ils font désormais partie de la représentation d'un corps amélioré, choisi et individuel.

13 Selon ses moyens économiques, car certains n'ont plus que leur corps ${ }^{31}$, l'hybride identitaire va incarner les nouvelles propriétés de notre être corporel : en équipant le corps de nouveaux matériaux, produits et technologiques, cette liberté de l'hybridité implique un engagement renouvelable par l'action sur le corps. S'hybrider, comme avec le pod d'Existenz de David Cronemberg, c'est se brancher sur une interaction corporelle qui vient modifier ses coordonnées esthésiologiques, ses processus mentaux et ses capacités motrices. La chirurgie esthétique propose des implants et autres infiltrations pour modifier de manière provisoire ou définitive l'image de son corps; l'entretien de son corps utilise des produits naturels ou artificiels (jusqu'au dopage) pour transformer la masse musculaire et améliorer les performances. L'hybridité fait ressentir en nous l'effet révélateur de l'interaction environnementale car elle s'incarne à l'occasion d'un événement.

14 En pouvant changer ses hybrides et prothèses, l'individu conserve une liberté d'initiative et a l'impression de pouvoir devenir un autre à chaque nouvelle hybridation. L'hybride ne se conserve pas dans l'être, il est en devenir par la mobilité, la fluctuation et la variation des états. Là où Rimbaud affirmait que je est un autre, 
l'hybride propose un devenir indéfini car l'opération peut se renouveler selon le désir et les possibilités offertes par le progrès biotechnologiques. Plus qu'une individualisation, l'hybride identitaire dessine sur le corps lui-même une personnalisation de la matière

Ce corps mutant ${ }^{32}$ ses représentations et créant ses normes n'est plus seulement une manière de travailler son apparence corporelle d'un corps pour soi ${ }^{33}$ mais une action par corps afin de dessiner ses modes incarnés de subjectivation. La subjectivation hybridée est le résultat de la personnalisation par le tatouage, le piercing, les implants, les prothèses, les compléments alimentaires et les médicaments. Système sémiotique, l'hybride identitaire se montre aux autres en se différenciant d'eux par un corps extraordinaire et artificiel. Le corps biotechnologique modifie les coordonnées subjectives en s'inventant son monde corporel. Le glissement de faire une mutation à être hybride dépend des pratiques corporelles de l'identité. Simple mutant, l'état corporel est seulement transformé de manière provisoire ou au cours d'une évolution biopsychique comme celle du passage de l'enfance à l'adolescence.

Le corps n'est pas une prothèse extérieure à nous-même, il se produit lui-même au moyen de ses hybridations avec son environnement; l'hybridité performe le soi corporel par un travail, un jeu et un exercice qui actualisent de nouvelles possibilités d'être jusque là inédites lors de l'ontogenèse et interdites par les normes. Le vivant n'arrête pas de produire de l'être dans le corps en réalisant des capacités par sa plasticité matérielle, la variation étant ainsi obtenue par déprogrammation ou reprogrammation ${ }^{34}$. Ce corps-devenir fait advenir en s'hybridant de nouvelles étapes de réalisation du soi qui ne sont jamais un soi-même.

Un toucher mixte

Cette inventivité fait reculer non seulement les représentations incorporées mais utilise la plasticité de la matière en réalisant de nouvelles constructions matérielles et symboliques. L'assignation de chaque être humain « autour de l'appareil reproducteur externe $»^{35}$, femme ou mâle, conduit à une séparation des sexes, des genres et des fonctions sociales. L'hybridation produit de nouvelles constructions mixtes par l'appropriation des techniques qui dénaturalise les fonctions, les objets et les modes relationnels. La difficulté de se libérer de la nature tient aux poids des représentations exaltant la domination masculine par " un programme social de perception incorporé $»^{36}$ qui s'applique au corps lui-même dans sa réalité biologique. L'hybridation, comprise comme incorporation d'habitus, est un moyen de reproduction politique des représentations et hiérarchies fonctionnelles construites par une culture donnée.

Mais l'hybridation suppose aussi que pour être hybride, il faut que l'être corporel soit double, à la fois michat-michien à l'intérieur d'un même corps et non plus dans un corps qui serait premier et un second qui serait l'être authentique du sujet corporel. L'authenticité de l'hybride est dans son être double et multiple plutôt que dans une évolution de l'individu dans son existence. En devenant hybride, l'être lui-même est hybridée. Le fait de s'hybrider vient prouver que le corps est une matière plastique : le biologique n'est pas un déterminisme naturel mais une interaction dynamique entre le corps et son environnement.

S'il y a des nouveaux modèles de la subjectivité corporelle, il faut admettre :

1. Que la subjectivité corporelle existait sous des modèles aujourd'hui dépassés. 


\section{NOTES}

1. M. Maffesoli, 1993, La contemplation du monde. Figures du style communautaire, Biblio Essais, p. 42.

2. Op. cit., p. 71.

3. Frédéric Ploton, 2004, Sex toys, Ed.Tana.

4. B. Préciado, 2001, Contra-sexuel, Paris, Ed. Balland

5. M.H. Bourcier, 2005, Sex politics. Queer Zones 2, Ed. la fabrique.

6. Mike Davis, 1998, Au-delà de Blade Runner, Los Angeles et l'imagination du désastre, Paris, ed. Allia, 2006, p. 7-27.

7. David Fontaine, 2006, No sex last year, Arte edition.

8. Florence Aubenas, 2005, La Méprise. L'affaire d'Outreau, Paris, Le seuil.

9. Dominique Baudis, 2005, Face à la calomnie, Press Pocket.

10. Catherine Deschamps, 2006, Le sexe et l'argent des trottoirs, Paris, Hachette.

11. Elsa Fayner, 2006, Violences, féminin pluriel. Les violences envers les femmes dans le monde contemporain, Ed. Librio. M. Hirigoyen, 2005, Femmes sous emprises. Les ressorts de la violence dans le couple, Paris, Oh Ed.

12. Gisèle Dambuyant-Wargny, 2006, Quand on n'a plus que son corps, Paris, Armand Colin. 
13. Anne Benoit, 2004, Un Spa chez soi : Week-ends de soins pour le corps et l'esprit, Paris, ed Marabout.

14. B. Andrieu, 2007, Le soin de toucher, Paris, Belles lettres. Préface David le Breton.

15. D. Le Breton, 2006, Le toucher de l'autre, La saveur du monde, Paris, ed. Métailié, p. 219-244.

16. Fionna Harrold, 2001, Massage : L'art de soigner le corps par le toucher, Paris,

Ed. Tredaniel, 2006. Marie Borrel, 2006, Le guide du massage au quotidien : l'harmonie du corps et de l'esprit entre vos mains, Ed. Solar.

17. Liz Wilde, 2006, Home Spa, De Stress, Ryland Peters\&Small

18. Anne Hopper, 2005, Massage érotique : Enrichissez vos relations intimes grâce au pouvoir du toucher, Ed Contre-dires.

19. M. Muller, 2005, Frappe-moi, Paris, ed. Blanche.

20. Axterdam, 2005, Osez le bondage, Paris, ed. La Musardine, p. 9-13.

21. Frédéric Ploton, 2004, Sex toys, Ed. Tana, p. 6-7.

22. Jacques Serguine, 2005, Cruelle Zélande, Paris, ed. la Musardine, p. 119.

23. Yann Moix, 2004, Partouz, Le livre de Poche, p. 153.

24. Nelly Arcan, 2004, Folle, PointSeuil, p.75.

25. Toni Bentley, 2004, Ma reddition, Paris, La musardine, Ed. 2006.

26. Lucie Lux, 2006, Je ne m'ennuie jamais toute seule..., Paris, coll. Blanche.

27. Koren Shadmi, 2006, Cours intérieurs. La boîte d'aluminum, p. 50-61.

28. Chantal Thomas, 2004, Le contrat, Souffrir, Payot, 2006, p. 95-124.

29. Fabienne Godet, 2006, Saufle respect que je vous dois

30. B. Andrieu, 1999, Un corps à soi. Critique du masochisme, Ed. InterUniversitaires.

31. G. Dambuyant-Wargny, 2006, Quand on a plus que son corps, Paris, Armand Colin, p.37.

32. Critique ${ }^{\circ}$ Les Mutants, Juin-juillet 2006.

33. C. Bromberger, ed., 2005, Un corps pour soi, Paris, P.U.F.

34. Catherine Malabou, 2006, Les régénérés : cellules souches, thérapie génique, clonage, Critique, ${ }^{\circ} 709-710$ Mutants, p. 529-540, ici p. 540.

35. Colette Guillaumin, 1992, Le corps construit, Sexe, race et Pratique du pouvoir. L'idée de Nature, Ed Côté-femmes, p. 117.

36. Pierre Bourdieu, 1998, La domination masculine, Paris, ed. seuil, p. 16.

37. Jean Luc Nancy, 2006, Toucher, dans B. Andrieu ed., Le dictionnaire du corps en SHS, Paris, ed. CNRS.

\section{RÉSUMÉS}

Si le toucher est à l'origine même de la relation thérapeutique, le toucher est devenu un nouveau style de communication sociale et communautaire du soi. Le style tactile participe d'une transmutation des valeurs par un autre rapport à l'altérité : dans une société concrète, d'action en commun "l'autre est celui que je touche et avec lequel je fais quelque chose qui me touche".

If the Touch is the original cause of therapeutic relation, the touch is nox a communicate style in social and communaty dimensions of self. The touch style is a transmutation of values in our 
relation with the alterity : in concrete relation and comune action, the other personn is a touch relation and a affective relation.

\section{AUTEUR}

\section{BERNARD ANDRIEU}

Bernard Andrieu : Professeur, Dir.Accorps Equipe Associée à l'UMR 7117 CNRS

Epistémologie du corps et des pratiques corporelles (Université Henri Poincaré/ Faculté du sport/ Nancy 1). Archives Poincaré et au GDR 3222 Anthropologie des représentations corporelles. bernard.andrieu@wanadoo.fr http://www.staps.uhp-nancy.fr/bernard/index.htm http://leblogducorps.canalblog.com 